\title{
Changing the course of schizophrenia - predictors of treatment outcome revisited
}

R Emsley, MB ChB, MMed, FCPsych, MD

P Oosthuizen, MB ChB, MMed, MD

D Niehaus, MB ChB, MMed, PhD

L Koen, MB ChB, MMed

B Chiliza, MB ChB, FCPsych

Department of Psychiatry, Stellenbosch University, Tygerberg, W Cape

Multiple factors play a role in determining the outcome of schizophrenia. However, the role of these factors is poorly understood, and research findings so far have been inconclusive and sometimes contradictory. Various demographic and baseline clinical factors have been reported to be associated with treatment outcome. Also, early symptom reduction after initiation of antipsychotic therapy is closely related to later treatment response. However, associations as such do not necessarily imply predictive value, and none of these factors can be regarded as clinically useful in predicting treatment outcome. This article discusses selected aspects of treatment outcome and its prediction in schizophrenia, focusing particularly on early treatment response, ethnicity, neurological soft signs, and the predictive value of a discriminant functional analysis model utilising a combination of putative predictors. Such a model holds promise, and it is to be hoped that future refinements will lead to a clinically useful model for predicting outcome.

Schizophrenia is an incapacitating brain disease, having been described as arguably the worst illness affecting humankind - costly in personal, social and financial terms.' It imposes a disproportionately large economic burden on patients and their families, health care systems and society, because of its early onset, devastating effects, and usually lifelong course. ${ }^{2}$

Antipsychotics were introduced to clinical practice about 50 years ago, and have proved to be very effective in treating acute psychotic symptoms. However, in the long term the picture is very different, and the overall outcome of schizophrenia is poor. ${ }^{3}$ In the majority of cases the illness is characterised by frequent relapses and rehospitalisations, ${ }^{4}$ and severe social and occupational impairment. ${ }^{3}$ Patients are frequently marginalised from society, unemployed ${ }^{5}$ and at risk for suicide. ${ }^{6}$

\section{Factors influencing treatment outcome}

Multiple factors may play a role in determining the outcome of the illness. For example, some patients are refractory to treatment from the outset, while others appear to acquire treatment refractoriness at some stage for reasons that are poorly understood. ' In many cases, non- or partial adherence to medication may be responsible for a relapse, or for the persistence of residual psychotic symptoms. ${ }^{8}$ Also, the presence of co-morbid disorders such as depression and substance abuse may complicate treatment and contribute to a poor overall outcome. ${ }^{9}$

\section{Demographic and baseline clinical predictors of outcome}

The factors determining treatment response are poorly understood, and research findings so far have been inconclusive and sometimes contradictory. Among other factors, a poorer response has been associated with male gender, history of obstetric complications, more severe positive symptoms, poorer attention at baseline, the development of parkinsonism during antipsychotic treatment, ${ }^{10}$ extrapyramidal symptoms (EPSs) before antipsychotic exposure, ${ }^{11}$ neurological soft signs, ${ }^{12}$ cognitive impairment ${ }^{13}$ and prolonged duration of untreated psychosis (DUP). ${ }^{14}$ However, associations as such do not necessarily imply predictive value, and none of these factors can be regarded as clinically useful in forecasting treatment outcome. ${ }^{15}$

\section{Early symptom reduction and treatment response}

An alternative approach has recently produced promising results - early symptom reduction within days of initiation of antipsychotic treatment appears to closely parallel later treatment response, suggesting that this could be a useful predictor of outcome, either alone or in combination with 
other variables. While an association between early treatment response and treatment outcome has long been recognised, ${ }^{16-23}$ it was not until recently that this topic has been studied in any detail. A meta-analysis ${ }^{24}$ of the data of numerous clinical trials challenged the long-held belief that antipsychotic medication has a delayed onset of action, with evidence showing that the largest proportion of the response actually occurs within the first week of treatment. Also, in a double-blind, placebo-controlled study ${ }^{25}$ involving 311 acutely ill patients with schizophrenia who were treated with $10 \mathrm{mg}$ of olanzapine intramuscularly, $7.5 \mathrm{mg}$ of haloperidol intramuscularly, or intramuscular placebo, the treatment groups showed greater reduction of symptoms, including core psychotic symptoms, within the first 24 hours. In another study with important implications, Correll et al. ${ }^{15}$ investigated the predictive value of early symptom changes 1 week after initiation of treatment and found that early non-improvement $1<20 \%$ reduction in Brief Pscyhiatric Rating Scale (BPRS) total score at 1 week) predicted non-response at 4 weeks in $100 \%$ of cases, suggesting that treatment refractoriness may already be identifiable after 1 week. The latter study was conducted in chronic schizophrenics with acute exacerbation of symptoms. We were interested in investigating whether this was also the case with first exposure to antipsychotic treatment - i.e. in firstepisode schizophrenia. Utilising data from a large ( $N=522)$ multinational, randomised, double-blinded trial comparing risperidone and haloperidol, ${ }^{2}$ we determined the time to clinical response (defined as $\geq 20 \%$ reduction from baseline in symptoms) in first-episode schizophrenia. A clinical response was achieved in $76 \%$ of subjects. Of these, $23.4 \%, 23.4 \%$, $18.6 \%$ and $12.6 \%$ responded by weeks 1, 2, 3 and 4, respectively. However, $25 \%$ did not respond until after the 4th week, and 11\% after the 8th week. It would therefore appear that, with first exposure to antipsychotic medication, while more patients respond within the first 2 weeks, the time to response varies widely and a subset of patients take much longer. ${ }^{26}$ These apparent differences in treatment response between first- and multi-episode patients have important implications for future practice guidelines when suggesting the duration of a treatment trial of antipsychotic medication.

\section{Ethnicity and outcome}

Ethnicity and culture are thought to substantially influence the clinical presentation, treatment response and overall outcome of schizophrenia. The emerging field of ethnopsychopharmacology has become a focus of considerable attention in recent years. While psychotropic drugs appear to be effective across cultural and ethnic boundaries, ${ }^{27}$ it is increasingly recognised that cross-cultural or crossethnic variations in responses to psychotropic agents do occur. ${ }^{28}$ Ethno-specific polymorphisms in genes that govern pharmacokinetic and pharmacodynamic properties of psychotropic drugs have been characterised, and could explain some of these ethnic variations. However, there are many other possible explanations for the observed differences. For example, factors such as body mass, diet, nutritional status, substance abuse, and co-morbid medical conditions are important, as well as social issues such as family support, accessibility of health services, and community attitudes towards health services. ${ }^{29}$

The clinical course of schizophrenia varies across cultures, with the outcome in developing countries generally being regarded as more favourable, ${ }^{30}$ although the evidence is not conclusive. ${ }^{31}$ Various explanations for this phenomenon have been proposed. For example, certain personal dynamics within the patient's family, particularly retention of the extended family, have been suggested as protective factors. ${ }^{32}$ Another possibility is that the illness expresses itself differently in certain ethnic groups. However, this is unlikely as studies have generally failed to show significant cross-cultural differences in core psychotic features. On the contrary, studies of the symptom structure of schizophrenia report them to be remarkably similar across cultures. ${ }^{33}$

Most published studies reporting ethnic differences in antipsychotic treatment have been retrospective chart reviews. While these studies have consistently reported racial disparities in the dose, route of delivery loral versus depot) and class of antipsychotic medication (conventional versus new-generation), reasons for these discrepancies are essentially speculative. African Americans are more likely than Caucasians to receive higher antipsychotic doses; ${ }^{34}$ they are also more likely to be prescribed long-acting depot formulations of antipsychotics, ${ }^{35}$ and are less likely than whites to receive new-generation antipsychotics. ${ }^{36}$ On the other hand, Asian Americans are more likely to receive lower doses of antipsychotics than Caucasians, ${ }^{37}$ and are reported to have higher plasma concentrations of antipsychotics. ${ }^{38}$

While the finding that African Americans are more likely to be prescribed higher doses and to receive depot antipsychotics and conventional rather than second-generation antipsychotics suggests a greater degree of refractoriness to treatment, the opposite may in fact be the case. We conducted a study in which we investigated the racial differences in 6-week outcome in randomised, controlled trials among the three 
most prevalent racial groups in South Africa (blacks, whites and mixed descent). ${ }^{39}$ We found that whites had the poorest response to acute antipsychotic treatment, while the mixeddescent and black patients responded significantly better ( $p=0.0001$ and $p=0.005$, respectively). But, we also found that baseline psychopathology scores for the black and mixed-descent groups were significantly higher than those for whites, possibly as a result of delayed help-seeking. ${ }^{39}$ Racial bias in health services remains a significant problem in many countries, including South Africa ${ }^{40}$ and the USA. ${ }^{41}$

The new-generation antipsychotics have substantially changed the way we treat patients with schizophrenia. But because of their much greater acquisition costs, patients in lower-income countries, particularly in Africa, Latin America, Asia and the Pacific, are being denied access to these treatments. ${ }^{42}$ This is of concern, and raises important human rights issues as the gap between the levels of psychiatric care in developed and developing countries continues to widen. ${ }^{43}$ Considering the evidence for superior efficacy and acceptability of the new-generation antipsychotics compared with their older counterparts, poverty becomes a risk factor for poorer outcome in schizophrenia.

\section{Neurological 'soft' signs and treatment outcome}

Subtle neurological abnormalities are more common in patients with schizophrenia than in healthy controls $44-50$ or patients with other psychotic disorders, ${ }^{50}$ and they are generally stable over time. ${ }^{51-52}$ These so-called neurological 'soft' signs (NSSs) are present at an early stage in the illness ${ }^{49,50,53-55}$ and are considered to be nonspecific markers of neurodevelopmental abnormality. ${ }^{56}$ Alternatively, it has been proposed that NSSs are secondary to psychiatric symptoms such as impaired attention, or even to the sideeffects of antipsychotic medication. ${ }^{57}$ NSSs have been associated with poor treatment outcome, ${ }^{12}$ although their predictive power has not been formally assessed. In a study conducted at our centre in a sample of 66 largely medicationnaïve first-episode subjects, ${ }^{58}$ while the NSS total scores did not change significantly over time, some tests were related to longer duration of untreated psychosis, and showed a tendency to improve as psychiatric symptoms resolved. Also, tests involving motor sequencing tasks were highly significant predictors of the later development of tardive dyskinesia. ${ }^{58}$ This finding is of potential importance in outcome prediction, as an association has been suggested between tardive dyskinesia and refractoriness in schizophrenia. ${ }^{59}$

\section{Assessing the clinical value of predictors of outcome}

Since the introduction of the first effective pharmacological treatments for schizophrenia, researchers have attempted to identify factors that could predict the outcome of treatment. ${ }^{60}$ There would be considerable benefits if it were possible to predict treatment outcome reliably. For example, it would allow clinicians to avoid unnecessary persistence with ineffectual treatment before attempting alternative strategies. This in turn would reduce the risk of accruing morbidity, the development of side-effects, the duration of hospitalisation, the level of care required, the amounts of concomitant medication prescribed, and the overall costs incurred. It would also help patients and therapists to set realistic and attainable goals in rehabilitation programmes. Clearly, therefore, the availability of reliable and easy-to-use outcome predictors would assist clinicians in improving long-term outcome, thereby changing the course of the illness.

Unfortunately, little progress has been made in identifying clinically meaningful predictors of treatment outcome, beyond identifying those baseline socio-demographic and clinical features that are associated with a better or poorer outcome in some, but not all studies. The inconsistencies in research findings are at least in part due to differences in methodologies employed in the various studies. ${ }^{3}$ For example, the nature of the sample populations, treatments applied, duration of the assessment period, and evaluation instruments employed differ considerably across studies. In addition, the outcome measures vary. For example, for many years treatment trials assessing the efficacy of antipsychotics have used the degree of symptom reduction from baseline to endpoint as the primary outcome measure. Other studies have defined specific outcome criteria, including treatment response (e.g. 20\% improvement in psychopathology scores from baseline), ${ }^{61}$ relapse rates ${ }^{62}$ or remission. ${ }^{63}$ Unfortunately, once again different criteria have been applied to define these measures.

In the assessment of outcome in schizophrenia our focus has changed over the years. This is to be expected as advances in treatment and management have emerged and a greater awareness of optimising outcome has developed. Thus, in the pre-antipsychotic era treatment was aimed largely 
at containment and avoidance of self harm. The advent of conventional antipsychotics allowed clinicians to focus attention on the reduction of acute symptoms. The availablity of the second-generation of antipsychotics resulted in clinicians striving for greater efficacy in treating positive symptoms, as well as a broader spectrum of efficacy regarding negative, cognitive and mood symptoms, and reduction of the sideeffect burden. Later, with accumulating long-term study evidence emphasising the chronic, relapsing nature of the illness, and particularly owing to the emergence of evidence linking the recurrence of psychotic symptoms with progression of the illness, attention focused on relapse prevention as a measure of outcome. ${ }^{61,62}$ Finally, attention is now shifting to the achievement of sustained resolution of symptoms (i.e. remission), in the hope that this will translate into better overall functioning, quality of life and autonomy.

Recently, in an attempt to improve and standardise the assessment of treatment outcome, a Remission in Schizophrenia Working Group proposed operational criteria defining remission in schizophrenia. ${ }^{64}$ These criteria define remission according to a threshold of severity of selected rating scale items rather than percentage improvements from a particular baseline. The rating scale items were selected on the basis of their representing the core features of the illness. They were derived from the 3 major symptom domains (negative, psychosis and disorganised) identified by means of factor analysis, and the 5 criteria specified in DSM-IV for a diagnosis of schizophrenia. Corresponding items on the most commonly used existing schizophrenia rating scales (Positive and Negative Symptom Scale (PANSS), Brief Psychiatric Rating Scale (BPRS), Scale for the Assessment of Positive Symptoms (SAPS) and Scale for the Assessment of Negative Symptoms (SANS)| were selected. The proposed criteria define remission as absent, borderline or mild symptom intensity level, where such symptoms do not influence an individual's behaviour. An additional requirement is that these criteria must have been met for a minimum duration of 6 months. While these proposed criteria may not be ideal, and have yet to be tested for their external validity, they represent a point of departure from which later refinements can be made. It is likely that the usefulness of these criteria will be assed in various research settings.

With this in mind, we applied these criteria to a sample of 57 subjects with first-episode psychosis who participated in a prospective study over 24 months, and evaluated various potential predictors of outcome. ${ }^{65}$ The primary aim of our study was to identify any baseline and early treatment variables that could be useful to clinicians in predicting remission and non-remission. We evaluated various demographic, baseline clinical and early treatment response variables for their power to predict remission or non-remission by means of a discriminant analysis model. We found that while $70 \%$ of the sample managed to meet the cross-sectional remission criteria for symptom reduction at some point in the study, only $40 \%$ managed to achieve the full criteria when the 6 month duration was applied. Also, only $33 \%$ were able to maintain their remission status to completion of the trial. The discriminant analysis model identified 4 variables that could correctly predict $80 \%$ of remitters and $82 \%$ of non-remitters. These predictor variables were: early clinical response 16 weeks), NSSs, DUP and the presence of depressive signs at baseline. Based on these and other findings, the following items may be useful in predicting patients who are likely to respond poorly to standard antipsychotic therapy: less than $20 \%$ improvement from baseline in PANSS total scores at 6 weeks, DUP > 1 year, presence of NSSs, and absence of depressive symptoms at baseline.

Future research is likely to refine the predictive capacity of demographic and clinical variables. However, early identification of poor responders to standard antipsychotic therapy alone is not sufficient. At the same time, new developments in the treatment of refractory patients will hopefully offer these patients other options in addition to the use of clozapine.

\section{References}

1. Aronson SM. Cost-effectiveness and quality of life in psychosis: the pharmacoeconomics of risperidone. Clin Ther 1997; 19: 139-147

2. Glazer WM, Johnstone BM. Pharmacoeconomic evaluation of antipsychotic therapy for schizophrenia. J Clin Psychiatry 1997; 58: Suppl 10, 50-54.

3. Robinson DG, Woerner MG McMeniman M, Mendelowitz A, Bilder RM Symptomatic and functional recovery from a first episode of schizophrenia or schizoaffective disorder. Am J Psychiatry 2004; 161: 473-479

4. Weiden PJ, Olfson M. Cost of relapse in schizophrenia. Schizophr Bull 1995; 21 : 419-429.

5. Mechanic D, Blider S, McAlpine DD. Employing persons with serious mental illness Health Aff (Millwood) 2002; 21: 242-253

6. Meltzer HY. Suicide in schizophrenia: risk factors and clozapine treatment. J Clin Psychiatry 1998; 59: Suppl 3, 15-20.

7. Lieberman JA, Alvir JM, Koreen A, et al. Psychobiologic correlates of treatment response in schizophrenia. Neuropsychopharmacology 1996; 14: Suppl 3, 13S21S.

8. Keith SJ, Pani L, Nick B, et al. Practical application of pharmacotherapy with longacting risperidone for patients with schizophrenia. Psychiatr Serv 2004; 55: $997-$ 1005

9. Gupta S, Hendricks S, Kenkel AM, Bhatia SC, Haffke EA. Relapse in schizophrenia: is there a relationship to substance abuse? Schizophr Res 1996; 20: 153-156.

10. Robinson DG, Woerner MG, Alvir JM et al. Predictors of treatment response from a first episode of schizophrenia or schizoaffective disorder. Am J Psychiatry 1999; 156: 544-549

11. Chatteriee A, Chakos M, Koreen A, et al. Prevalence and clinical correlates of extrapyramidal signs and spontaneous dyskinesia in never-medicated schizophrenic patients. Am J Psychiatry 1995; 152: 1724-1729. 
12. Arango $C$, Kirkpatrick B, Buchanan RW. Neurological signs and the heterogeneity of schizophrenia. Am J Psychiatry 2000; 157: 560-565

13. Moritz S, Krausz M, Gottwalz E, et al. Cognitive dysfunction at baseline predicts symptomatic 1-year outcome in first-episode schizophrenics. Psychopathology 2000; 33(1): 48-51.

14. Ucok A, Polat A, Genc A, Cakiotar S, Turan N. Duration of untreated psychosis may predict acute treatment response in first-episode schizophrenia. J Psychiatr Res 2004 38: 163-168.

15. Correll CU, Malhotra AK, Kaushik S, McMeniman M, Kane JM. Early prediction of antipsychotic response in schizophrenia. Am J Psychiatry 2003; 160: 2063-2065

16. Ribeiro SC, Tandon R, Ricoy R, Mazzara C, Craig KA, Greden JF. Clinical predictors of 1-year outcome in schizophrenia. Psychopathology 1992; 25: 331 1-334.

17. Rifkin A, Doddi S, Karajgi B, Wachspress M, Boppana V. Neuroleptic treatment and prediction of response. Psychopharmacol Bull 1988; 24: 169-171.

18. Zemlan FP, Thienhaus OJ, Garver DL. Length of psychiatric hospitalization and prediction of antipsychotic response. Prog Neuropsychopharmacol Biol Psychiatry 1990; 14: 13-24

19. Stern RG, Kahn RS, Harvey PD, Amin F, Apter S, Hirschowitz J. Early response to haloperidol treatment in chronic schizophrenia. Schizophr Res 1993; 10: 165-171.

20. Bartko G, Maylath E, Herczeg I. Comparative study of schizophrenic patients relapsed on and off medication. Psychiatry Res 1987; 22: 221-227.

21. May PR, Van Putten T, Yale C. Predicting outcome of antipsychotic drug treatment from early response. Am J Psychiatry 1980; 137: 1088-1089.

22. Gaebel W, Pietzcker A, Ulrich G, Schley J, Muller-Oerlinghausen B. Predictors of neuroleptic treatment response in acute schizophrenia: results of a treatment study with perazine. Pharmacopsychiatry 1988; 21: 384-386.

23. Breier A, Schreiber JL, Dyer J, Pickar D. National Institute of Mental Health longitudinal study of chronic schizophrenia. Prognosis and predictors of outcome. Arch Gen Psychiatry 1991; 48:239-246.

24. Agid O, Kapur S, Arenovich T, Zipursky R. Delayed-onset hypothesis of antipsychotic action: A hypothesis tested and rejected. Arch Gen Psychiatry 2003; 60: 1228 1235.

25. Kapur S, Arenovich T, Agid O, Zipursky R, Lindborg S, Jones B. Evidence for onset of antipsychotic effects within the first 24 hours of treatment. Am J Psychiatry 2005; 162: 939-946

26. Emsley R, Rabinowitz J, Medori R. Time course for antipsychotic treatment response in first-episode schizophrenia. Am J Psychiatry 2006; 163: 743-745

27. Lin KM, Anderson D, Poland RE. Ethnicity and psychopharmacology. Bridging the gap. Psychiatr Clin North Am 1995: 18: 635-647.

28. Poolsup N, Li Wan PA, Knight TL. Pharmacogenetics and psychopharmacotherapy. J Clin Pharm Ther 2000; 25: 197-220

29. Ruiz P. Ethnicity and Psychopharmacology.Review of Psychiatry. Washington, DC: American Psychiatric Press, 2000.

30. Kulhara P. Outcome of schizophrenia: some transcultural observations with particular reference to developing countries. Eur Arch Psychiatry Clin Neurosci 1994; 244: 227-235

31. Edgerton RB, Cohen A. Culture and schizophrenia: the DOSMD challenge. Br J Psychiatry 1994; 164: 222-231.

32. Marcolin MA. The prognosis of schizophrenia across cultures. Ethn Dis 1991; 1: 99104.

33. Emsley RA, Niehaus DJ, Mbanga NI, et al. The factor structure for positive and negative symptoms in South African Xhosa patients with schizophrenia. Schizophr Res 2001; 47: 149-157.

34. Diaz FJ, de Leon J. Excessive antipsychotic dosing in 2 U.S. State hospitals. J Clin Psychiatry 2002; 63: 998-1003.

35. Kreyenbuhl J, Zito JM, Buchanan RW, Soeken KL, Lehman AF. Racial disparity in the pharmacological management of schizophrenia. Schizophr Bull 2003; 29: 183 193.

36. Kuno E, Rothbard $A B$. Racial disparities in antipsychotic prescription patterns for patients with schizophrenia. Am J Psychiatry 2002; 159: 567-572.

37. Collazo Y, Tam R, Sramek J, Herrera J. Neuroleptic dosing in Hispanic and Asian inpatients with schizophrenia. Mt Sinai J Med 1996; 63: 31 0-313.

38. Lin KM, Poland RE, Lau J. Haloperidol and prolactin concentrations in Asians and Caucasians. J Clin Psychopharmacol 1988; 8: 195-201.

39. Emsley RA, Roberts MC, Rataemane $S$, et al. Ethnicity and treatment response in schizophrenia: a comparison of 3 ethnic groups. J Clin Psychiatry 2002; 63(1): 9-14.

40. Swartz L. The politics of black patients' identity: ward-rounds on the 'black side' of a South African psychiatric hospital. Cult Med Psychiatry 1991; 15: $217-244$.
41. Lawson WB. Issues in pharmacotherapy for African Americans. In: Ruiz P, ed. Review of Psychiatry. Washington, DC: American Psychiatric Press, 2000: 37-53.

42. Emsley RA, Oosthuizen PP, Joubert AF, Hawkridge SM, Stein DJ. Treatment of schizophrenia in low-income countries. Int J Neuropsychopharmcol 1999; 2: 321 325.

43. Sartorius N, Emsley RA. Psychiatry and technological advances: implications for developing countries. Lancet 2000; 356: 2090-2092

44. Heinrichs DW, Buchanan RW. Significance and meaning of neurological signs in schizophrenia. Am J Psychiatry 1988; 145(1): 11-18.

45. Buchanan RW, Heinrichs DW. The Neurological Evaluation Scale (NES): a structured instrument for the assessment of neurological signs in schizophrenia. Psychiatry Res 1989: 27: 335-350

46. Mohr F, Hubmann W, Cohen R, et al. Neurological soft signs in schizophrenia: assessment and correlates. Eur Arch Psychiatry Clin Neurosci 1996; 246: 240248.

47. Egan MF, Hyde TM, Bonomo JB, et al. Relative risk of neurological signs in siblings of patients with schizophrenia. Am J Psychiatry 200 1; 158: 1827-1834.

48. Yazici AH, Demir B, Yazici KM, Gogus A. Neurological soft signs in schizophrenic patients and their nonpsychotic siblings. Schizophr Res 2002; 58: 241-246.

49. Shibre T, Kebede D, Alem A, et al. Neurological soft signs (NSS) in 200 treatment naive cases with schizophrenia: a community-based study in a rural setting. Nord J Psychiatry 2002; 56: 425-431.

50. Keshavan MS, Sanders RD, Sweeney JA, et al. Diagnostic specificity and neuroanatomical validity of neurological abnormalities in first-episode psychoses. Am J Psychiatry 2003; 160: 1298-1304

51. Chen EY, Lam LC, Chen RY, Nguyen DG. Neurological signs, age, and illness duration in schizophrenia. J Nerv Ment Dis 1996; 184: 339-345.

52. Marcus J, Hans SL, Lewow E, Wilkinson L, Burack CM. Neurological findings in high-risk children: childhood assessment and 5-year followup. Schizophr Bull 1985; 11(1): 85-100

53. Gupta S, Andreasen NC, Arndt S, et al. Neurological soft signs in neuroleptic-naive and neuroleptic-treated schizophrenic patients and in normal comparison subjects. Am J Psychiatry 1995; 152: 191-196.

54. Venkatasubramanian $G$, Latha V, Gangadhar BN, et al. Neurological soft signs in never-treated schizophrenia. Acta Psychiatr Scand 2003; 108: 144-146.

55. Whitty $P$, Clarke $M$, Browne $S$, et al. Prospective evaluation of neurological soft signs in first-episode schziophrenia in relation to psychopathology: state versus trait phenomena. Psychol Med 2003; 33: 1479-1484

56. Griffiths TD, Sigmundsson T, Takei N, Rowe D, Murray RM. Neurological abnormalities in familial and sporadic schizophrenia. Brain 1998; 121: Part 2 191-203.

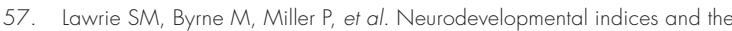
development of psychotic symptoms in subjects at high risk of schizophrenia. $\mathrm{Br}$ J Psychiatry 2001; 178: 524-530

58. Emsley R, Turner J, Oosthuizen P, Carr J. Neurological abnormalities in first episode schizophrenia: temporal stability and clinical and outcome correlates. Schizophr Res 2005: 75: 35-44

59. Chakos MH, Alvir JM, Woerner MG, et al. Incidence and correlates of tardive dyskinesia in first episode of schizophrenia. Arch Gen Psychiatry 1996; 53: 31 3319

60. Gaebel W. Prediction of response to acute neuroleptic treatment in schizophrenia. Int Clin Psychopharmacol 1996; 11 : Suppl 2, 47-54

61. Schooler N, Rabinowitz 」, Davidson M, Emsley R, Harvey P, Kopala L, McGorry P Van Hove I, Eerdekens M, Swyzen W, De Smedt G, and the Early Psychosis Global Working Group. Risperidone and haloperidol in first episode psychosis: a long-term randomized trial. Am J Psychiatry 2005; 162: 947-953.

62. Csernansky JG, Mahmoud R, Brenner R. A comparison of risperidone and haloperidol for the prevention of relapse in patients with schizophrenia. NEnglJ Med 2002; 346(1): 16-22

63. Lieberman J. Jody D Geisler $\mathrm{S}$, et al Time course and biologic correlates of treatment response in first-episode schizophrenia. Arch Gen Psychiatry 1993; 50: 369-376.

64. Andreasen N, Carpenter W, Kane J, Lasser R, Marder SR, Weinberger DR. Remission in Schizophrenia: Proposed Criteria and Rationale for Consensus. Am J Psychiatry 2005; 162: 44 1-449

65. Emsley R, Oosthuizen P, Kidd M, Koen L, Niehaus D, Turner HJ. Remission in schizophrenia: predictor variables and symptom improvement patterns. J Clin Psychiatry 2006; 67 (1 1): 1707-1712 